\title{
THE RESOLVENT OF A CLOSED TRANSFORMATION
}

\author{
A. E. TAYLOR
}

1. Introduction. In reading over Chapter IV of Stone's book, Linear Transformations in Hilbert Space, I was impressed by the fact that a number of the results obtained are valid for any complex Banach space. This generality does not always appear at once evident, and it may be worth while to explain briefly.

The most interesting and important fact which underlies the material is that the resolvent of a closed distributive* transformation depends analytically on a parameter $\lambda$. This dependence is made precise in Stone's work with the aid of the inner product of Hilbert space; but this is not necessary, for it is known that the fundamental portions of the classical theory of analytic functions remain valid in complex Banach spaces. $\dagger$ In particular, Liouville's theorem admits a valid generalization. Thus we are able to prove that the spectrum of a (continuous) linear transformation whose domain is the whole space $E$ is not empty. We shall now turn to the details.

2. Preliminaries. We use $E$ to denote a complex Banach space; $T$ will denote a distributive (additive, homogeneous) transformation, with domain and range both in $E$. We then write $T_{\lambda}=T-\lambda I$, and $T_{\lambda}^{-1}$ will denote the inverse of $T_{\lambda}$ when it exists. Here $\lambda$ is a complex number, and $I$ the identity transformation. We recall that a transformation admits an inverse if and only if it sets up a one to one correspondence between its domain and its range. When $T$ is distributive, the necessary and sufficient condition that $T_{\lambda}^{-1}$ exist is that $T_{\lambda} f=0$ imply $f=0$. The set of values of $\lambda$ for which $T_{\lambda}^{-1}$ is linear, with domain everywhere dense in $E$, is called the resolvent set of $T$. All other values of $\lambda$ constitute the spectrum of $T$.

3. Discussion of the resolvent. We prove the following theorem:

THEOREM 1. If $T_{\lambda_{0}^{-1}}^{-1}$ exists and is linear, then $T_{\lambda^{-1}}^{-1}$ exists and is linear for each $\lambda$ such that $\left|\lambda-\lambda_{0}\right|<1 / M_{\lambda_{0}}$, where $M_{\lambda_{0}}$ is the modulus of $T_{\lambda_{0}}^{-1}$.

* We use distributive where Stone uses linear, preferring to reserve the latter term for continuous distributive transformations. For the definition of a closed transformation see Stone, loc. cit., p. 38.

$\dagger$ For the independent variable a complex number this was pointed out by Wiener, Fundamenta Mathematicae, vol. 4 (1923). For the general case see A. E. Taylor, Comptes Rendus, vol. 203 (1936), pp. 1228-1230, and a forthcoming paper in the Annali della Reale Scuola Normale di Pisa; also L. M. Graves, this Bulletin, vol. 41 (1935), pp. 651-653. 
Proof. Let $f$ be the domain of $T$. Then $T_{\lambda} f=T_{\lambda_{0}} f-\left(\lambda-\lambda_{0}\right) f$. But since $T_{\lambda_{0}}{ }^{-1}$ is linear, $\|f\| \leqq M_{\lambda_{0}}\left\|T_{\lambda_{0}} f\right\|$, and hence if $\left|\lambda-\lambda_{0}\right|<1 / M_{\lambda_{0}}$, $T_{\lambda} f=0$ implies $f=0$, so that $T_{\lambda}^{-1}$ exists. Also, since

$$
\|T f-\lambda f\| \geqq\left\|T_{\lambda_{0}} f\right\|-\left|\lambda-\lambda_{0}\right|\|f\| \geqq\left(\frac{1}{M_{\lambda_{0}}}-\left|\lambda-\lambda_{0}\right|\right)\|f\|,
$$

we conclude that $T_{\lambda}^{-1}$ is bounded, and hence linear. Its modulus does not exceed $M_{\lambda_{0}} /\left(1-M_{\lambda_{0}}\left|\lambda-\lambda_{0}\right|\right)$.

THEOREM 2. If $T$ is closed, and if its resolvent set is not empty, the domain of $T_{\lambda}^{-1}$ is the whole space $E$ when $\lambda$ is in the resolvent set.

The proof of this offers no difficulties, and we omit it. The family of linear transformations $T_{\lambda}^{-1}$ is called the resolvent. We denote it by $R_{\lambda}$.

The next theorem is a direct carry over from Stone (Theorem 4.10 , p. 137). The proof given by Stone is valid in the present case, and the reader is referred to it.

THEOREM 3. If the resolvent $R_{\lambda}$ of a closed distributive transformation $T$ exists, then $R_{\lambda}-R_{\mu} \equiv(\lambda-\mu) R_{\lambda} R_{\mu}$ throughout $E$ for each pair of values $\lambda, \mu$ in the resolvent set, and $R_{\lambda} f=0$ implies $f=0$.

Conversely, if $X_{\lambda}$ is a family of linear transformations with domain $E$, defined for each $\lambda$ in a set $\Sigma$, such that

$$
X_{\lambda}-X_{\mu} \equiv(\lambda-\mu) X_{\lambda} X_{\mu}
$$

for each $\lambda, \mu$ in $\Sigma$; and if $X_{\lambda} f=0$ implies $f=0$ for at least one $\lambda$ in $\Sigma$, then there exists a unique closed, distributive transformation $T$ whose resolvent exists and coincides with $X_{\lambda}$ for every $\lambda$ in $\Sigma$.

The functional equation (1) is striking. It suggests at once a "law of the mean" for $X_{\lambda} f$, and in a sense is just that. We shall consider some further conclusions which may be drawn from (1) under suitable hypotheses.

Theorem 4. Let $X_{\lambda}$ be a linear transformation with domain $E$ for each $\lambda$ in an open set $\Sigma$ of the complex plane; further, let $X_{\lambda} f$ be continuous in $\Sigma$ for each $f$ in $E$, and suppose that the functional equation (1) is satisfied when $\lambda, \mu$ are in $\Sigma$. Then $X_{\lambda} f$ is analytic* in $\Sigma$ for each $f$ in $E$, and in the neighborhood of each point of $\Sigma$ admits an expansion in the form

* In the sense indicated by Wiener. See the references in an earlier footnote. 


$$
X_{\lambda} f=\sum_{n=0}^{\infty}\left(\lambda-\lambda_{0}\right)^{n} X_{\lambda_{0}}^{n+1} f
$$

where $\lambda_{0}$ is in $\Sigma$ and $\left|\lambda-\lambda_{0}\right|$ is sufficiently small.

Proof. We first prove that $X_{\lambda} f$ admits a derivative with respect to $\lambda$ at each point of $\Sigma$. The form of (1) suggests that this derivative is $X_{\lambda}^{2} f$; this surmise is verified by the inequality

$$
\left\|\frac{X_{\lambda} f-X_{\lambda_{0}} f}{\lambda-\lambda_{0}}-X_{\lambda_{0}}^{2} f\right\|=\left\|X_{\lambda_{0}}\left(X_{\lambda} f-X_{\lambda_{0}} f\right)\right\| \leqq M_{\lambda_{0}}\left\|X_{\lambda} f-X_{\lambda_{0}} f\right\|,
$$

where $M_{\lambda_{0}}$ is the modulus of $X_{\lambda_{0}}$, since $X_{\lambda} f$ is continuous with respect to $\lambda$ (it is easily seen that $X_{\lambda} X_{\mu} \equiv X_{\mu} X_{\lambda}$ ). It follows from the general theory of abstract-valued analytic functions that $X_{\lambda} f$ is analytic in $\Sigma$. In particular, it admits derivatives of all orders in $\Sigma$, and is expansible in a Taylor series. In order to show that this expansion about a point $\lambda=\lambda_{0}$ has the form (2), we shall prove that the derivatives of $X_{\lambda} f$ are given by the formula

$$
\left[X_{\lambda} f\right]^{(n)} \equiv \frac{d^{n}}{d \lambda^{n}}\left[X_{\lambda} f\right]=n ! X_{\lambda}^{n+1} f
$$

This has already been established for $n=1$. We proceed by induction, assuming the truth of (3) with $n$ replaced by $n-1$. Then

$$
\begin{aligned}
& \left\|\frac{\left[X_{\lambda} f\right]^{(n-1)}-\left[X_{\lambda_{0}} f\right]^{(n-1)}}{\lambda-\lambda_{0}}-n ! X_{\lambda_{0}}^{n+1} f\right\| \\
& \quad=\left\|(n-1) ! \frac{X_{\lambda}^{n} f-X_{\lambda_{0}}^{n} f}{\lambda-\lambda_{0}}-n ! X_{\lambda_{0}}^{n+1} f\right\| \\
& \quad=\left\|(n-1) ! \frac{X_{\lambda}-X_{\lambda_{0}}}{\lambda-\lambda_{0}}\left(X_{\lambda}^{n-1}+X_{\lambda}^{n-2} X_{\lambda_{0}}+\cdots+X_{\lambda_{0}}^{n-1}\right) f-n ! X_{\lambda_{0}}^{n+1} f\right\| \\
& \quad=\left\|(n-1) ! X_{\lambda_{0}} X_{\lambda}\left(X_{\lambda}^{n-1}+X_{\lambda}^{n-2} X_{\lambda_{0}}+\cdots+X_{\lambda_{0}}^{n-1}\right) f-n ! X_{\lambda_{0}}^{n+1} f\right\| \\
& \quad \leqq(n-1) ! M_{\lambda_{0}}\left\|\left(X_{\lambda}^{n}+X_{\lambda}^{n-1} X_{\lambda_{0}}+\cdots+X_{\lambda} X_{\lambda_{0}}^{n-1}\right) f-n X_{\lambda_{0}}^{n} f\right\| .
\end{aligned}
$$

But the expression inside the last norm sign tends to zero as $\lambda \rightarrow \lambda_{0}$, by virtue of the continuity of $X_{\lambda}$, and so the induction is completed. The continuity of the iterates of $X_{\lambda}$, and therefore of the expression inside the norm, is deduced from the fact that $X_{\lambda} f$, being linear in $f$, is continuous in $\lambda$ and $f$ together.*

* This is a theorem of Kerner, Studia Mathematica, vol. 3 (1931), p. 159. Formula (3) could also be established by use of some theorems about Fréchet differentials. For the above direct proof I am indebted to the referee. 
From (3) and the fact that $\Sigma$ is an open set we can infer the validity of (2) for some region $\left|\lambda-\lambda_{0}\right|<\rho$. This completes the proof of Theorem 4.

However, it is apparent that (2) converges and defines a linear transformation with domain $E$ whenever $\left|\lambda-\lambda_{0}\right|<1 / M_{\lambda_{0}}$. If this range of values was not originally included in $\Sigma$, the domain of definition of $X_{\lambda}$ may be extended, and it is easy to see that (1) will continue to be satisfied.

The foregoing considerations suggest at once the nature of the resolvent of $T$.

THEOREM 5. Let $T$ be a closed, distributive transformation whose resolvent exists. Then the resolvent set $\Sigma$ is an open set, and the resolvent $R_{\lambda} f$ is an analytic function of $\lambda$ in $\Sigma$. If $M_{\lambda}$ is the modulus of $R_{\lambda}$, $\Sigma$ is such that with $\lambda_{0}$ it contains all points $\lambda$ such that $\left|\lambda-\lambda_{0}\right|<1 / M_{\lambda_{0}}$. $R_{\lambda}$ is then given by the expansion

$$
R_{\lambda} f=\sum_{n=0}^{\infty}\left(\lambda-\lambda_{0}\right)^{n} R_{\lambda_{0}}^{n+1} f
$$

and its modulus satisfies the inequality

$$
M_{\lambda} \leqq \frac{M_{\lambda_{0}}}{1-\left|\lambda-\lambda_{0}\right| M_{\lambda_{0}}} .
$$

Proof. Since $\Sigma$ is non-empty, it contains a point $\lambda_{0}$, and $R_{\lambda_{0}}$ is a linear transformation with domain $E$ (Theorem 2). Let us define a family of transformations $X_{\lambda} f$ by the series on the right in equation (4). It is clear that $X_{\lambda} f$ will be linear, with domain $E$, when $\left|\lambda-\lambda_{0}\right|<1 / M_{\lambda_{0}}$. Next we identify $X_{\lambda}$ with $T_{\lambda}^{-1}$ when $\left|\lambda-\lambda_{0}\right|<1 / M_{\lambda_{0}}$. The calculations are identical with those in Stone, pages 140-141, and will be omitted. By definition, then, the resolvent $R_{\lambda}$ of $T$ exists and coincides with $X_{\lambda}$ when $\left|\lambda-\lambda_{0}\right|<1 / M_{\lambda_{0}}$. The resolvent set is accordingly open. Equation (4) now gives $R_{\lambda} f$ as a power series with abstract coefficients; it is therefore analytic as a function of $\lambda$. This result may be obtained without further resort to the theory of abstract power series by appealing to Theorem 4 , since $R_{\lambda} f$ is now evidently continuous as a function of $\lambda$, and satisfies the functional equation (1), by Theorem 3. The inequality (5) has already been noted in Theorem 1. Theorem 5 is thus proved.

4. The resolvent of a linear transformation. We state now another theorem: 
Theorem 6. If $T$ is linear, with domain $E$ and modulus $C$, the resolvent set contains all $\lambda$ such that $|\lambda|>C$, and for these values

$$
R_{\lambda} f=-\sum_{n=1}^{\infty} \frac{1}{\lambda^{n}} T^{n-1} f .
$$

Thus all the singularities of $R_{\lambda} f$ lie in the finite part of the plane. In particular $R_{\lambda} f$ has at least one singularity, that is, the spectrum of $T$ is not empty.

The proof of (6) is well known. The last assertion of the theorem follows from Liouville's theorem (for abstract analytic functions), since from (6) we have

$$
\left\|R_{\lambda} f\right\| \leqq \frac{\|f\|}{|\lambda|-C}, \quad|\lambda|>C .
$$

If the spectrum of $T$ were empty, $R_{\lambda} f$ ( $f$ fixed) would be analytic over the entire plane and finite at infinity. Then $R_{\lambda} f$ would be a constant, with value 0 , for all $f$, since $\lim _{|\lambda| \rightarrow \infty}\left\|R_{\lambda} f\right\|=0$. This is impossible. (We exclude, of course, the trivial case where $E$ consists of the zero element alone.)

It would be interesting to know other relationships between the nature of $T$ and the singularities of its resolvent.

California Institute of Technology 